\title{
Application of multiple-group latent growth model to determine the effect of shift work on body mass index among petrochemical industries staff
}

\author{
Maedeh Amini ${ }^{1}$, Farid Zayeri*2 \\ Received: 16 Nov 2018 \\ Published: 12 Oct 2019
}

\section{Abstract}

Background: Shift work can be defined as work activity scheduled in which employees work outside standard daytime hours. Some reports have shown that shift work is associated with several health problems. The main objective of this research was to explore the effect of shift work on body mass index (BMI) over time among Iranian petroleum industry staff.

Methods: This longitudinal study sample was consisted of 3,686 (1,872 day workers and 1,814 shift workers) petrochemical industries staff in Mahshahr, southwest of Iran, from 2012 to 2015. The weight and height of these staff were measured using standard techniques and equipment. Multiple-group latent growth curve modeling was used to determine the effect of shift working on BMI trajectories over this period of time via Mplus software, version 6.0.

Results: The mean $( \pm \mathrm{SD})$ age of the shift workers and day workers were $38.96( \pm 8.36)$ and $43.33( \pm 8.35)$ years, respectively. Findings from the modeling approach showed that the slope of BMI in shift workers on average was $0.14 \mathrm{~kg} / \mathrm{m} 2 \mathrm{greater}$ than the day workers in years under study $(\mathrm{p}<0.001)$.

Conclusion: The findings of the current study suggest that shift work can be considered as a potential risk indicator for higher weight gain in petrochemical staff. Hence, health policy-makers should organize health screening programs and periodical checkups to find high-risk staff and control unhealthy lifestyle factors in these industries.

Keywords: Shift work, Body mass index, Longitudinal, Latent, Growth curve modeling

Conflicts of Interest: None declared

Funding: None

*This work has been published under CC BY-NC-SA 1.0 license.

Copyright $($ Iran University of Medical Sciences

Cite this article as: Amini M, Zayeri F. Application of multiple-group latent growth model to determine the effect of shift work on body mass index among petrochemical industries staff. Med J Islam Repub Iran. 2019 (12 Oct);33:109. https://doi.org/10.47176/mjiri.33.109

\section{Introduction}

Obesity has been noted as one of the epidemic public health problems in many societies (1). It is one of the leading causes of diet-related death and disability, with about 2.8 million people dying every year throughout the world. The relative participation in economic development, speedy urbanization, subsequent lifestyle changes, and also heredity factors can be thought as the main reasons for the growing epidemic of obesity (2).

Generally, according to common definitions for adults,

\footnotetext{
Corresponding author: Dr Farid Zayeri, $f$ zayeri@sbmu.ac.ir

1. Department of Biostatistics, Faculty of Medical Sciences, Tarbiat Modares University, Tehran, Iran

2. Proteomics Research Center and Department of Biostatistics, Faculty of Paramedical Sciences, Shahid Beheshti University of Medical Sciences, Tehran, Iran
}

a Body Mass Index (BMI) between 25 and $29.99 \mathrm{~kg} / \mathrm{m}^{2}$ and a BMI of 30 or higher are considered as overweight and obese, respectively (3). Being overweight and obese can lead to adverse metabolic effects on blood pressure, cholesterol, cancer, triglycerides, and insulin resistance. Based on a report from the World Health Organization (WHO) in 2015, there were 2.3 billion overweight and 700 million obese people in the world, and the prevalence of this health problem is still increasing (4).

$\uparrow$ What is "already known" in this topic:

- Some studies have indicated that the prevalence of overweight and obese people among shift workers is higher than day workers.

$\rightarrow$ What this article adds:

- The statistical tool in this article enables us to compare the baseline levels and trends of BMI in between day and shift workers.

- In the present study, we found that the shift work schedule can cause overweight among the petrochemical staff. 
Nowadays, in many countries particularly industrialized countries, due to factors such as competing with other rivals, optimal use of resources and need for higher levels of production, producers tend to increase the working time to 24 hours $(5,6)$. This leads to a need for working outside normal hours, which is referred to as shift work. In other words, shift work can be defined as working on a schedule outside traditional hours, including evening or night, early morning shifts, and any irregular or rotating schedules (7). In different industries, shift work schedules are common in many sectors and they can vary from one workplace to another (5). Based on previously published literature, shift work is one of the potential factors which may increase the risk for development of many deleterious effects on one's health such as digestive diseases, heart disease, epilepsy, substance abuse, asthma, occupational accidents, circadian rhythm disorders, gastrointestinal problems, psychological disorders, coronary heart disease, certain cancers, metabolic syndrome, chronic fatigue as well as disturbance of social, family life, performance deterioration, and impairment in sleep quality (7-9).

Shift work schedule is common around the world. For instance, in Asia, it is broadly applied in China, Malaysia, and Korea. According to a report by US Bureau of Labor Statistics in 2007, more than 21 million people usually worked at least partially outside the daytime shift range (10). In such circumstances, health promotion programs in the workplace such as enhanced access to opportunities for physical activity combined with health education can be a potential avenue for preventing or controlling the mentioned shift work disorders (11).

Several prior epidemiological studies have explored the relationship between higher BMI and shift work (11-15). Nonetheless, the association between shift work and body weight has not been determined conclusively, and it is still controversial. A review of previous literature identified that the prevalence of overweight and obese people among shift workers is higher than day workers $(6,16,17)$. In contrast, some other studies have indicated an opposite relationship (18). Regarding this we decided to conduct the present study on petrochemical staff of Mahshahr city, southwest of Iran, to assess the relationship between shift work and BMI in a large sample over time using more advanced statistical techniques. To do this, we utilized a longitudinal data based on a four-year period. To model the effect of shift working on repeated measures of BMI, we applied a multiple-group latent growth curve methodology. This statistical tool enables us to compare the baseline levels and trends of BMI in different groups under study (in our study, day workers and shift workers) over a specific period of time.

\section{Methods}

\section{Dataset}

In this longitudinal study, the data from 3686 staff of petrochemical industries in Bandar Mahshahr city (southwest of Iran) was investigated from 2012 to 2015 . According to the periodic (annual) examinations, all of the staff who referred to the center of occupational medicine for assessing some anthropometric measures and other im- portant outcomes such as blood tests were included in the study. Therefore, the gathered data was related to all the participants, and there was no need to sampling.

In this research, the main outcome under study was the body mass index (BMI) of these subjects during the described period of time. To calculate BMI, the weight and height of each subject were measured annually by standardized techniques and equipment. The height of each individual was measured using a safe metal ruler (without shoes), and the weight was measured in light clothing using calibrated scales.

As mentioned before, the main aim of this paper was to determine the effect of shift working on repeated measures of BMI. To do this, the staff was divided into two groups; shift work (staff with routine clockwise rotation plan, i.e., four morning shifts, four evening shifts and four night shifts) and day work (staff with a routine working time from 8:00 am to 4:00 pm).

\section{Statistical Analysis}

The latent growth curve (LGC) method represents a broad class of statistical methods that involve several advantages associated with longitudinal data: (a) provides enhanced statistical power, (b) permits examination of intra-individual (i.e., change within individuals across time) change over time as well as interindividual (i.e., individual differences in change across time) variability in intra-individual change $(19,20)$.

In the present paper, the fitting model for the outcome variable (BMI) includes two steps. First, an unconditional LGC model (LGCM) (i.e., without predictors) was fitted to investigate the overall pattern of change in the reported outcome. Furthermore, latent growth curve model and the two latent variables were used to estimate baseline BMI and its changes over 4 years (intercept and slope). Second, the shift schedule as a binary predictor variable $(1=$ day work and $2=$ shift work) was added to assess the direct effect on the growth trajectories. This model is referred to multiple-group LGC modeling to examine growth patterns in BMI across shift groups. For each of the models, the factor loadings related to the intercept were all fixed at 1 , while the loadings related to the slope factors are fixed at values which represent the expected pattern of change proportional to the time of each measurement occasion.

The general latent growth curve model, for the repeated outcome measure $y_{t i}$ (i.e. BMI) of individual $i$ at occasion $\mathrm{t}$, as a function of two latent variables $\left(\eta_{0 i}\right.$ and $\left.\eta_{1 i}\right)$ can be described as:

$$
\begin{aligned}
y_{t i} & =\lambda_{0 t} \eta_{0 i}+\lambda_{1 t} \eta_{1 i}+\varepsilon_{t i} \\
\quad \mathrm{i} & =1,2, \ldots, 3686 ; \mathrm{t}=2012,2013,2014,2015 \\
\eta_{0 i} & =\alpha_{0}+\zeta_{0 i} \\
\eta_{1 i} & =\alpha_{1}+\zeta_{1 i}
\end{aligned}
$$

Where Equation 1 is the within subject model, in which the latent growth factors $\eta_{0 i}$ and $\eta_{1 i}$ expressed as functions of latent means $\left(\alpha_{0}\right.$ and $\left.\alpha_{1}\right)$ and individual deviations away from those means, respectively and the $\lambda_{t} \mathrm{~s}$ are 
time scores. Equations 2 and 3 are the between subject models, where $\eta_{0}$ denotes the model estimated overall mean level of the initial BMI, $\eta_{1}$ represents the average rate of BMI change over time. Moreover, the $\zeta$ residuals, representing between subject variations in regard to the BMI growth trajectory, are referred to as random effects. The residual term $\varepsilon_{t i}$ in equation 1 is error term at time t, representing time specific influence of the $i^{\text {th }}$ individual $(20,21)$.

Before stages 1 and 2 for investigating the shape of the trajectory of BMI over time (linear or nonlinear trends), we fitted two models in which the slope factors were fixed as follows: $0,1,2,3$ (linear) and $0,1,4,9$ (quadratic). For each model, adequate model fit was concluded if the root mean square error of approximate (RMSEA) was under 0.05 , the comparative fit index (CFI) and the TuckerLewis Index (TLI) were over 0.9, and lower AIC.

In the univariate analysis, the mean age differences between shift and day workers were evaluated by independent-sample t-test. All P values (two-sided) less than 0.05 were considered as statistically significant. The data were analyzed using descriptive methods by SPSS 20.0, and several models were fitted in Mplus version 6.0.

\section{Results}

A total of 3686 petrochemical personnel were included in this study. Table 1 displays the characteristics of the sample, separately for day and shift workers. Among these participants, $1,814(49.2 \%)$ subjects were shift work and $1,872(50.8 \%)$ were day work. The number of males (3574) and females (112) included in the present research accounted for about $97.0 \%$ and $3.0 \%$, respectively. The mean $( \pm \mathrm{SD})$ age of these staff was $41.18( \pm 8.63)$ years, ranged from 24 to 66 years. Likewise, the mean $( \pm \mathrm{SD})$ ages of the shift and day workers were $38.96( \pm 8.35)$ years old and $43.33( \pm 8.35)$ years old, respectively.

Primarily, for evaluating the shape of the mean trend of BMI over time, the described fit indices were used. Table 2 shows some of the goodness of fit indices. Comparing the fit indices revealed that the linear model (CFI $>0.99$,
TFI $>0.99$, RMSEA $<0.01)$ provided better fit to describe the pattern of change in petrochemical workers than the model with quadratic growth factors (CFI $=0.90$, $\mathrm{TFI}=0.88$, RMSEA=0.21). Consequently, in the first step of data analysis, a linear LGCM was fitted to the data from all of the personnel (including shift and day workers).

Table 3 represents the obtained estimates of LGC model. As shown, the overall mean BMI at the starting point of the study (intercept in the year 2012) was 26.10 $(p<0.001)$. Also, the overall average rate of change (slope) in the BMI was 0.26 , indicating the BMI of the staff increased significantly on average by 0.26 per year $(p<0.001)$. The significant variance of the estimated intercept displays that there were between-person differences in the baseline BMI values of the staff $(p<0.001)$. Also, the significant variance of the slope indicates that there was substantial variation in BMI trajectories among participants over the study period $(p<0.001)$. In addition, the significant negative correlation between the intercept and slope implies that workers with higher initial values of $\mathrm{BMI}$ had a less reduction in BMI over the 4-year period.

In the second stage of the modeling process, a multiple group LGCM was utilized for comparing the baseline levels (in 2012) and the trend of BMI changes in the day and shift workers over time. Figure 1 illustrates the path diagram of this model. The parameter estimates of multiplegroup LGCM are provided in Table 4 . With respect to the results of the multi-group LGCM fitting, one can conclude that the day workers had a slightly higher initial level of BMI than the shift workers (mean Intercept $=26.22$ vs. $25.90)$ and this difference was significant $(p<0.001)$. In addition, comparing the estimated slopes from this model tells us that the trend of BMI in the shift workers had a steeper slope than the day workers (mean slope $=0.347$ vs. $0.203, p<0.001$ ). In other words, the annual trend of average BMI in the shift workers was about $0.14 \mathrm{~kg} / \mathrm{m}^{2}$ higher than the day workers. The findings also demonstrated that there were significant intercept factor variances among day workers and shift workers in BMI (variance of 15.28

Table 1. Demographic characteristics of the staff participated in the study according to shift schedule groups

\begin{tabular}{lccc}
\hline Variables & \multicolumn{2}{c}{ Shift Schedule } & Total \\
\cline { 2 - 4 } Gender & Shift & Day & $<0.001^{\mathrm{b}}$ \\
Female & $10(8.9)^{\mathrm{a}}$ & $102(91.1)$ & $112(100.0)$ \\
Male & $1804(50.5)$ & $1770(49.5)$ & $3574(100.0)$ \\
Age (Mean \pm SD) & $38.96 \pm 8.35$ & $43.33 \pm 8.35$ & $41.18 \pm 8.38$ \\
\hline
\end{tabular}

Abbreviation: SD, standard deviation; ${ }^{\mathrm{a}}$ No. $(\%),{ }^{\mathrm{b}}$ Chi-square test; ${ }^{\mathrm{c}}$ Independent sample t-test.

Table 2. Summary of the goodness of fit indices to assess best-fitting model and describe the trend of change in petrochemical staff

\begin{tabular}{lccccc}
\hline Alternative Models & AIC & CFI & TFI & RMSEA \\
\hline Total BMI Linear & 103973.150 & 1.000 & 1.000 & 0.000 & $<0.001$ \\
Total BMI quadratic & 104802.831 & 0.903 & 0.883 & 0.209 & $<0.001$ \\
\hline
\end{tabular}

104802.831

0.903

0.883

0.209

$<0.001$

Table 3. Parameter estimates from the unconditional latent growth curve modeling analysis of linear change in BMI over follow up

\begin{tabular}{lccc}
\hline Parameters & Path Est. & SE & \\
\hline Mean Intercept & 26.10 & 0.09 & \\
Mean Slope & 0.26 & 0.02 & $<0.001$ \\
Intercept Variance & 15.93 & 1.34 & $<0.001$ \\
Slope Variance & 1.27 & 0.33 & $<0.001$ \\
COV (Intercept, Slope) & -0.40 & 0.06 & $<0.001$ \\
\hline Note: All path estimates shown are unstandardized except for correlations between Intercept and Slope that is standardized
\end{tabular}




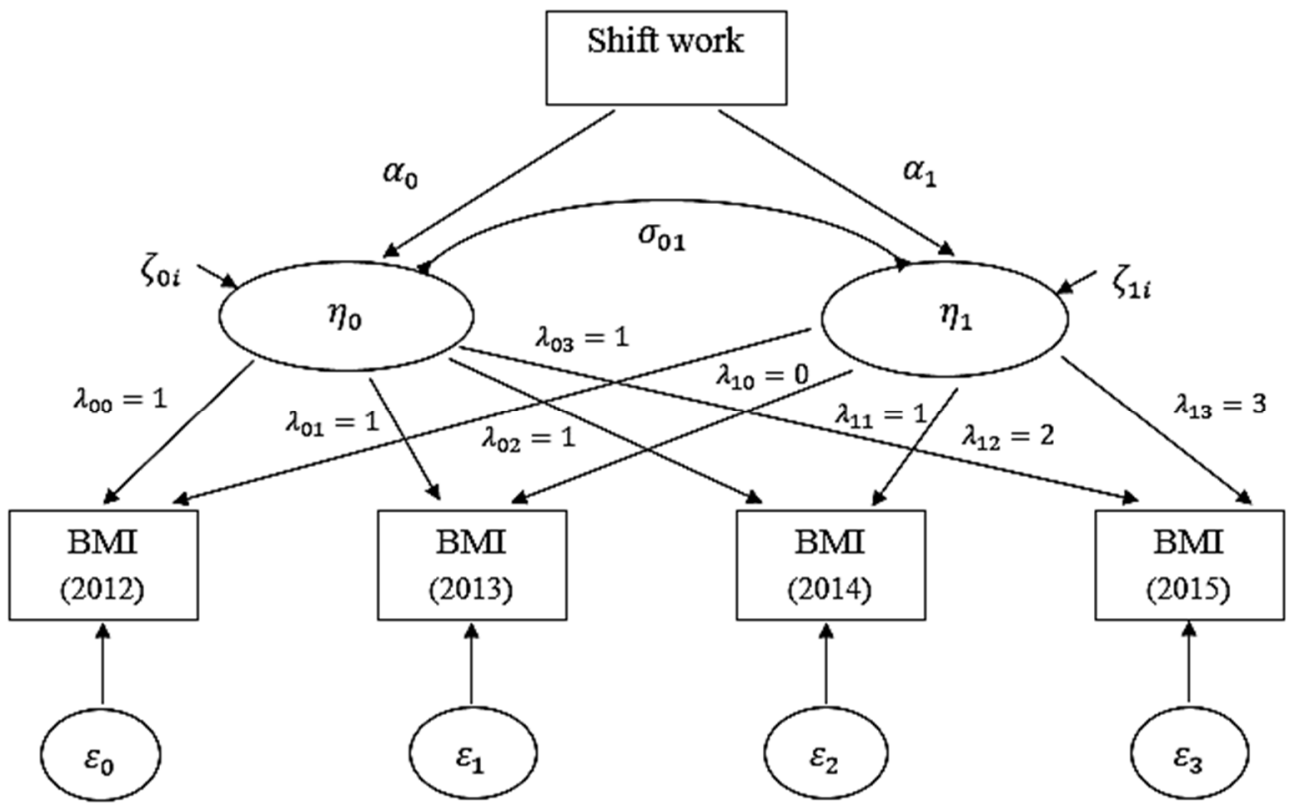

Fig. 1. The path diagram of multiple group LGC model for the BMI outcome over a four-year follow-up period

Table 4. The estimated path coefficients of BMI using a multiple-group latent growth curve model in the petrochemical staff across 4 time points

\begin{tabular}{lccc}
\hline Parameters & Path Est. & SE & \\
\hline Mean Intercept & & & $<0.001$ \\
Day workers (Group 1) & 26.222 & 0.096 & $<0.001$ \\
Shift workers (Group 2) & 25.903 & 0.124 & $<0.001$ \\
Mean Slope & & $<.203$ & $<0.001$ \\
Day workers (Group 1) & 0.347 & 0.020 & $<0.001$ \\
Shift workers (Group 2) & & 0.034 & $<0.001$ \\
Intercept Variance & 15.284 & 0.730 & 0.008 \\
Day workers (Group 1) & 16.384 & 2.034 & $<0.001$ \\
Shift workers (Group 2) & & 0.274 & $<0.001$ \\
Slope Variance & 1.733 & 0.476 & $<0.001$ \\
Day workers (Group 1) & & & \\
Shift workers (Group 2) & -0.328 & 0.050 & 0.073 \\
COV (Intercept, Slope) & -0.403 & & \\
Day workers (Group 1) & & \\
Shift workers (Group 2)
\end{tabular}

Note: All path estimates shown are unstandardized except for correlations between Intercept and Slope (standardized). Also we estimated the model simultaneously for day and shift workers without constraints on any of the parameters.

with $\mathrm{p}<0.001$ for day workers, and variance of 16.38 with $\mathrm{p}<0.001$ for shift workers). Likewise, the estimated significant variances for the slopes show remarkable variability in the trajectories of BMI values for the day and shift workers during the period under study (variance of 0.27

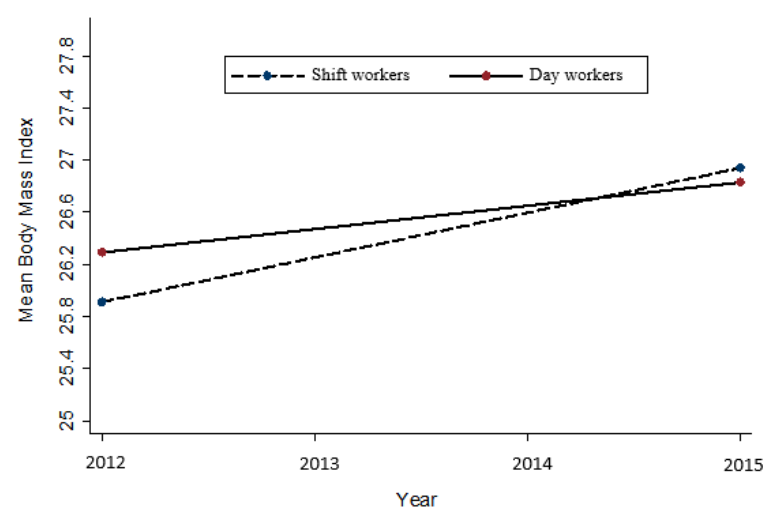

Fig. 2. Growth trajectories of BMI across schedule groups among staff in the period of four years based on estimates of multiple latent growth modeling parameters. with $\mathrm{p}=0.008$ for day workers, and variance of 1.73 with $\mathrm{p}<0.001$ for shift workers). Moreover, the smaller negative correlation for the shift workers indicates that the higher initial levels of BMI in the shift workers was related to a greater reduction in BMI over time than the day workers. Figure 2 displays the trend of estimated mean BMI, separately in the day and shift workers during years under study.

\section{Discussion}

According to recent investigations, shift workers constitute more than $20 \%$ of the whole working population in industrial countries. Previous epidemiological studies have led researchers to believe that shift work has emerged as a serious occupational risk factor for the employees and meet the operational needs of the organizations (22). The key question in the current study was whether shift work is associated with increased risk for higher BMI among petrochemical staff. To clarify this issue, multi-group LGC modeling was performed for examining the sample mean and individual differences in the 
initial levels and trajectories of BMI across shift work schedules.

Prior to conducting the multi-group LGC model, the LGCM was fitted to determine the total mean intercept (i.e., starting point) and mean slope of change in BMI among all personnel irrespective of working groups. The findings suggested that the overall mean level of the initial BMI for all staff was higher than $25 \mathrm{~kg} / \mathrm{m}^{2}(=26.10$ $\mathrm{kg} / \mathrm{m}^{2}$ ). Consequently, we concluded that the subjects were more likely to be at risk for being overweight. Factors like unhealthy lifestyle, low physical activity because of the sedentary nature of their work, socio-demographics, level of education, family income, and some behavior habits (e.g., smoking status) are causally linked to become overweight. This result is consistent with a number of similar published studies suggesting that overweight elicit a series of diseases, resulting in a public health problem for staff (23-27). Hence, there is an urgent need to improve awareness and also community-based multiple strategies to combat the increasing level of BMI across different industries personnel. Notably, the variance estimates for intercept and slope represented a significant degree of variability around the overall mean initial BMI and linear change in BMI over time. This variability implied that there was substantial unobserved heterogeneity between and within subjects owing to latent individuallevel factors.

In the second stage of the analysis, the baseline and trend of BMI of staff between the two groups of shift and day work were compared via multi-group LGC approach. In this review, the model identified that shift and day working were significantly positively associated with BMI at the baseline level and rate of change in BMI over time. Further, the findings revealed that the larger increase in BMI at the start of follow-up time (i.e., in 2012) occurred among the day workers (26.22 vs. 25.90). The reasons for the higher BMI in 2012 may be attributed to less time for physical activity, disruption of circadian rhythm, long working hours, and changes in eating behaviors among the day workers $(5,10)$. Moreover, a number of prospective and cross-sectional studies have noted that female and male gain weight as they age. Indeed, age as a factor may lead to increases in adiposity and concurrent reduction in energy consumption $(28,29)$. Based on results obtained in Table 1, the age can account for the differences at the baseline level of BMI between the day and shift workers, such that the mean age of the day workers (mean age= 43.33 years) was significantly higher compared with the mean age of the shift workers (mean age $=39.36$ years). Generally, this paper considers key findings from several studies. With regards to previous studies, our results are in agreement with only one report from Suwazono et al. in a longitudinal study (6) which discovered that the day workers compared to the shift workers have greater BMI at the baseline level due to worse habits where the association was significant. In contrast, other pieces of evidence implicated a greater increase in BMI among shift workers than day workers and also observed a significant relationship between BMI and shift working (30-34). For instance, a longitudinal study by Kazemi et al. had been shown that odds of being overweight or obese in shift workers was about 18 percent higher than the day workers (35). On the other hand, some cross-sectional studies have failed to demonstrate statistically significant differences between BMI and shift work schedule, which were in contradiction with our findings $(36,37)$.

Further, the present paper also clearly showed that the annual trend of average BMI in shift workers was comparatively 0.14 times greater than that of the day workers between 2012 and 2015. Accordingly, shift workers are at greater risk for higher levels of BMI due to some factors over time. Indeed, this finding could highlight that lifestyle characteristics and lack of regular exercise as potential mediators may relate directly to overweight among the shift workers (38). Also, working irregular hours in shift workers could be a leading cause of the increasing level of BMI. This irregularity has forced the staff to work against normal asleep and awake cycle and devote all or parts of night to work and day to rest. Hence, lack of balance in arrangement of working hours leads to fatigue and reduced physical activities. Accordingly, public health professionals should consider scheduling in the workplace, increase social awareness regarding the need for regularity in daily rhythms, including the sleep-wake cycle, and also an adequate opportunity for sleep for the shift workers. On the other hand, in this study, direct adjustment of factors was impossible, including age that can affect the association between shift work and BMI as a nonmodifiable risk factor. Age can mediate the effect of shift work pattern on BMI across the four-time period. Nevertheless, in latent growth model, without adjustment for age as a confounder, findings was not confounded; because based on reported of Table 1, the mean age of day workers was higher than shift workers and considering the relationship between age and weight, this difference should be more impact on day workers compared shift workers. The finding agrees with the report of Di Lorenzo et al. in a cross-sectional study on the association between shift work and BMI by multiple regression analysis among chemical industry workers. They found that BMI was significantly higher among shift workers independent of age and history of work covariates (31). However, in another cross-sectional study by Parkes, the increase in the level of BMI tended to be greater among the day-shift workers than among those working day-night shift schedules when modified for age, though the difference was not significant (39).

Furthermore, in this context, the random effects (variances of intercept and slope) were statistically significant for the groups of work schedule separately. The values of baseline at the start of follow-up time and rates of increase in BMI over time were not similar across the shift and day workers. Therefore, in our opinion, it seems possible that some unobserved genetic or environmental factors are responsible for individual differences at the initial level and trajectory of BMI during the years 2012 to 2015 .

There are a few longitudinal studies that have established the effect of shift work on the increased risk of obesity or weight gain over time. A longitudinal observational study of 469 nurses showed that weight gain was more 
prevalent among night workers compared to day-time workers during a five-year period (40) Morikawa et al. (33) have performed a longitudinal cohort study on 1529 Japanese male blue-collar workers in a sash and zipper factory over a period of 10 years. They revealed significantly elevated levels of BMI using a multiple regression model among shift workers. Finally, Suwazono and colleagues (6) conducted a 14-year follow up study between 1991 and 2005 on day and alternating workers at the time of their annual health checkups. Using pooled multiple logistic regression analysis, it was found that working at alternating shift was associated with a significantly higher rate of BMI during the study. However, among the mentioned longitudinal studies, the only two studies considering possible confounding factors were those of Morikawa et al. (33) and Suwazono et al. (6) who acquired significant difference in BMI levels between shift workers and day workers after adjusting for all potential confounders (e.g., age) in their model.

To the best of our knowledge, this is the first longitudinal study performed in Iran and other countries of the world for determining the effect of shift work on the trend of variation in the mean BMI across different groups. However, we suggest that researchers conduct additional studies using a multi-group LGC modeling approach to examine the longitudinal association between various BMI categories and different types of shift work schedule among petrochemical staff by adjusting for potential confounding factors.

Overall, two main advantages associated with the use of LGCM over competing methods which adopted approaches used to study change among outcome variables, such as analysis of covariance and multilevel modeling, can be drawn from this study. First, unlike similar longitudinal studies, the quality of our research findings was enhanced, because of using a proper analysis approach regarding the nature of data collection. Also, the model offers greater insight about individual differences. Secondly, the hypothesis about the shape of trajectories over time can be tested by this method. Moreover, the large sample size was another advantage of this research. On the other hand, the current study has also had several limitations that should be noted. First, in this study, the direct age adjustment, history of work, and other covariate variables in the shift workers were impossible. Second, the lack of exact information about the reason of increased BMI in shift workers compared to day workers. Third, we focused only on the staff in Mahshahr petrochemical company, Iran. Therefore, our results cannot be generalized to all petrochemical staff across Iran. As the last limitation, in this paper, the proportion of the female workforces was very low compared with their male counterparts.

\section{Conclusion}

In summary, the present paper found that the shift work schedule can cause overweight among the petrochemical staff. Accordingly, in order to improve the staff' health, educational programs including regular checkups for unhealthy lifestyle factors (e.g., diet and physical activity), adjustment the shift work schedule, and other aspects of working conditions appear to be vital.

\section{Acknowledgments}

The authors wish to express their utmost thanks to Dr. Hormoz Hassanzade for his permission to us to use the dataset for this research.

\section{Conflict of Interests}

The authors declare that they have no competing interests.

\section{References}

1. Alsaif MA, Hakim IA, Harris RB, Alduwaihy M, Al-Rubeaan K, AlNuaim AR, et al. Prevalence and risk factors of obesity and overweight in adult Saudi population. Nutr Res. 2002;22(11):124352.

2. Hou X, Jia W, Bao Y, Lu H, Jiang S, Zuo Y, et al. Risk factors for overweight and obesity, and changes in body mass index of Chinese adults in Shanghai. BMC Public Health. 2008;8(1):389.

3. Humayun A, Shah AS, Sultana R. Relation of hypertension with body mass index and age in male and female population of Peshawar, Pakistan. J Ayub Med Coll Abbottabad. 2009;21(3):63-5.

4. Ahmadi M, Moosazadeh M, Vardanjani HM-E, Dehghan A. Prevalence of obesity and overweight and their related factors among the adults of Mazandaran Province, Iran, in 2010. Electron Physician. 2014;6(4):955.

5. Khajehnasiri F, Mortazavi SB, Allameh AB, Akhondzadeh S. Serum Level of Cortisol, hsCRP and IL-6 in Depressed Rotational Shift Workers. Thrita. 2013;2(2):139-44.

6. Suwazono Y, Dochi M, Sakata K, Okubo Y, Oishi M, Tanaka K, et al. A Longitudinal Study on the Effect of Shift Work on Weight Gain in Male Japanese Workers. Obesity. 2008;16(8):1887-93.

7. Kivimäki M, Batty GD, Hublin C. Shift work as a risk factor for future type 2 diabetes: evidence, mechanisms, implications, and future research directions. PLoS Med. 2011;8(12):e1001138.

8. Choobineh A, Soltanzadeh A, Tabatabaee H, Jahangiri M. Health Effects Associated With Shift Work in 12-Hour Shift Schedule Among Iranian Petrochemical Employees. Int J Occup Saf Ergon. 2012;18(3):419-27.

9. Knutsson A, Alfredsson L, Karlsson B, Åkerstedt T, Fransson EI, Westerholm P, et al. Breast cancer among shift workers: results of the WOLF longitudinal cohort study. Scan J Work Environ Health. 2013;39(2):170-7.

10. Eberly R, Feldman H. Obesity and Shift Work in the General Population. Int J Allied Health Sci Pract. 2010;8(3):1-9.

11. Smith P, Fritschi L, Reid A, Mustard C. The relationship between shift work and body mass index among Canadian nurses. Appl Nurs Res. 2013;26(1):24-31.

12. Amani R, Gill T. Shiftworking, nutrition and obesity: implications for workforce health- a systematic review. Asia Pac J Clin Nutr. 2013;22(4):698-708.

13. Anderson L, Quinn T, Glanz K, Ramirez G, Kahwati L, Johnson D, et al. The effectiveness of worksite nutrition and physical activity interventions for controlling employee overweight and obesity- A systematic review. Am J Prev Med. 2009;37:340-57.

14. Schulte P, Wagner G, Ostry A, Blanciforti L, Cutlip R, Krajnak K. Work, obesity, and occupational safety and health. Am J Public Health. 2004;97:428-36.

15. Luckhaupt SE, Cohen MA, Li J, Calvert GM. Prevalence of obesity among US workers and associations with occupational factors. Am J Prev Med. 2014;46(3):237-48.

16. Antunes LC, Levandovski R, Dantas G, Caumo W, Hidalgo MP. Obesity and shift work: chronobiological aspects. Nutr Res Rev. 2010;23:155-68

17. Kim MJ, Son KH, Park HY, Choi DJ, Yoon CH, Lee HY, et al. Association between shift work and obesity among female nurses: Korean Nurses' Survey. BMC Public Health. 2013;13:1-8.

18. Dochi M, Sakata K, Oishi M, Tanaka K, Kobayashi E, Suwazono Y. Relationship between shift work and hypercholesterolemia in Japan. Scand J Work Environ Health. 2008;34(1):33-9.

19. Felt JM, Depaoli S, Tiemensma J. Latent growth curve models for biomarkers of the stress response. Front Neurosci. 2017;11:315. 
20. Diallo TMO, Morin AJS. Power of Latent Growth Curve Models to Detect Piecewise Linear Trajectories. Struct Equ Model: A Multidisciplin J. 2015;22(3):449-60.

21. Wang J, Wang X. The ABC's of LGM: An Introductory Guide to Latent Variable Growth Curve Modeling. Soc Personal Psychol Compass. 2009;3(6):979-91.

22. obinson D. A cross-sectional analysis of the association between night-only or rotating shift work and overweight/obesity among female nurses and midwives. J Occup Environ Med. 2012;54(7):83440.

23. Macagnan J, Pattussi MP, Canuto R, Henn RL, Fassa AG, Olinto MTA. Impact of nightshift work on overweight and abdominal obesity among workers of a poultry processing plant in southern Brazil. Chronobiol Int. 2012;29(3):336-43.

24. Peplonska B, Bukowska A, Sobala W. Association of rotating night shift work with BMI and abdominal obesity among nurses and midwives. PLoS One. 2015;10(7):e0133761.

25. Barbadoro P, Santarelli L, Croce N, Bracci M, Vincitorio D, Prospero E, et al. Rotating shift-work as an independent risk factor for overweight Italian workers: a cross-sectional study. PLoS One. 2013;8(5):e63289.

26. Buss J. Associations between obesity and stress and shift work among nurses. Workplace Health Saf. 2012;60(10):453-8.

27. Son M, Ye BJ, Kim JI, Kang S, Jung KY. Association between shift work and obesity according to body fat percentage in Korean wage workers: data from the fourth and the fifth Korea National Health and Nutrition Examination Survey (KNHANES 2008-2011). Ann Occup Environ Med. 2015;27(1):32.

28. Williams PT, Wood PD. The effects of changing exercise levels on weight and age-related weight gain. Int J Obes. 2006;30(3):543-51.

29. Van Amelsvoort L, Schouten E, Kok F. Duration of shiftwork related to body mass index and waist to hip ratio. Int J Obes Relat Meta Disord. 1999;23(9):973-8.

30. Chee HL, Kandiah M, Khalid M, Shamsuddin K, Jamaluddin J, Nordin N, et al. Body mass index and factors related to overweight among women workers in electronic factories in Peninsular Malaysia. Asia Pac J Clin Nutr. 2004;13(3):248-54.

31. Di Lorenzo L, De Pergola G, Zocchetti C, L'abbate N, Basso A, Pannacciulli N, et al. Effect of shift work on body mass index: results of a study performed in 319 glucose-tolerant men working in a Southern Italian industry. Int $\mathrm{J}$ Obes Relat Metab Disord. 2003;27(11):1353-8.

32. Ishizaki M, Morikawa Y, Nakagawa H, Honda R, Kawakami N, Haratani T, et al. The influence of work characteristics on body mass index and waist to hip ratio in Japanese employees. Ind Health. 2004;42(1):41-9.

33. Morikawa Y, Nakagawa H, Miura K, Soyama Y, Ishizaki M, Kido $\mathrm{T}$, et al. Effect of shift work on body mass index and metabolic parameters. Scand J Work Environ Health. 2007;33(1):45-50.

34. Ostry AS, Radi S, Louie AM, Anthony AD. Psychosocial and other working conditions in relation to body mass index in a representative sample of Australian workers. BMC Public Health. 2006;6:53.

35. Kazemi M, Abadi A, Zayeri F, Hassanzade H. Assessing the effect of shift work among petrochemical Industries staff at Mahshahr, Iran. Arch Adv Biosci. 2017;8(4):36-43.

36. Ghiasvand M, Heshmat R, Golpira R, Haghpanah V, Soleimani A, Shoushtarizadeh P, et al. Shift working and risk of lipid disorders: a cross-sectional study. Lipids Health Dis. 2006;5(1):1-9.

37. Karlsson BH, Knutsson AK, Lindahl BO, Alfredsson LS. Metabolic disturbances in male workers with rotating three-shift work. Results of the WOLF study. Int Arch Occup Environ Health. 2003;76(6):42430 .

38. Gomez-Parra M, Romero-Arrieta L, Vasquez-Trespalacios EM, Palacio-Jaramillo V, Valencia-Martinez A. Association between shift work and being overweight or obese among health care workers in a clinical setting in Medellin, Colombia. Work. 2016;55(3):635-42.

39. Parkes KR. Shift work and age as interactive predictors of body mass index among offshore workers. Scand J Work environ Health. 2002;28(1):64-71.

40. Niedhammer I, Lert F, Marne M. Prevalence of overweight and weight gain in relation to night work in a nurses' cohort. Int J Obes Relat Metab Disord. 1996;20(7):625-33. 